\title{
ON SOME NATURES OF THE EXCITATION AND DAMPING OF THE POLAR MOTION
}

\author{
NAOSUKE SEKIGUCHI
}

Tokyo Astronomical Observary, University of Tokyo, Mitaka, Tokyo, Japan

\begin{abstract}
Values of the Chandlerian period and the damping coefficient of the polar motion are calculated for every ten years from 1900.0 to 1970.0, using a method developed by Sekiguchi (1966). The damping coefficient is variable between 0.074 and $0.008(\mathrm{yr})^{-1}$ through this period, and correlative with the variation of the Chandlerian period, as Sekiguchi (1961) theorized.
\end{abstract}

1. The motion of the coordinates of the pole position $(x, y)$ is governed by equations

$$
\begin{aligned}
& \dot{x}+c(y-Y)+\kappa x=0, \\
& \dot{y}-c(x-X)+\kappa y=0,
\end{aligned}
$$

where $X$ and $Y$ are stochastic functions of time $t$, and ordinarily called excitation functions. $c$ is the angular velocity of the Chandlerian motion and $\kappa$ is the damping coefficient. Both quantities are considered as constants for time intervals of ten years or so, but they are assumed to be variable for a time interval longer than decade.

We assume that the mean values of the stochastic functions $X$ and $Y$ are zero, and their behaviour is stationary, that is, the two-dimensional auto-correlation function

$$
\mu_{Q}(s)=\langle X(t) X(t+s)+Y(t) Y(t+s)\rangle
$$

is independent of $t$.

From these assumptions, the author deduced (Sekiguchi, 1966) that the auto-correlation function of $x$ and $y$

$$
\mu_{q}(s)=\langle x(t) x(t+s)+y(t) y(t+s)\rangle
$$

is also independent of $t$, and satisfies the equation

$$
\mu_{q}(s)=\left(c^{2} / 2 \kappa\right) \int_{-\infty}^{\infty} \mu_{Q}(t) \cos c(t-s) e^{-\kappa|t-s|} \mathrm{d} t .
$$

2. If we assume that $\mu_{Q}(t)=0$ for sufficiently large values of $t$, we have

$$
\mu_{q}(s)=e^{-\kappa|s|}(A \cos c s+B \sin c|s|),
$$

where $A$ and $B$ are constants. As the functional form of $\mu_{q}(s)$ is obtainable from the observations, we can determine the values of the constants $c, \kappa, A$ and $B$, so as to fit the expression (5) to the calculated values as well as possible. In the course of this calculation, the secular part and the annual component are subtracted from the ob- 
TABLE I

\begin{tabular}{llllll} 
& \multicolumn{1}{c}{$\begin{array}{l}\kappa \\
(\mathrm{yr})^{-1}\end{array}$} & $\begin{array}{l}c \\
(\mathrm{yr})^{-1}\end{array}$ & $\begin{array}{l}A \\
\left(1^{\prime \prime}\right)^{2}\end{array}$ & $\begin{array}{l}B \\
\left(1^{\prime \prime}\right)^{2}\end{array}$ & $\begin{array}{l}2 \pi / c \\
=\text { Chandler's period } \\
(\mathrm{yr})\end{array}$ \\
\hline $1900-1910$ & +0.038 & 5.367 & +0.0254 & +0.0013 & 1.171 \\
$1910-1920$ & +0.017 & 5.221 & +0.0358 & -0.0010 & 1.203 \\
$1920-1930$ & +0.074 & 5.647 & +0.0076 & +0.0015 & 1.113 \\
$1930-1940$ & +0.067 & 5.352 & +0.0061 & -0.0015 & 1.174 \\
$1940-1950$ & +0.054 & 5.256 & +0.0302 & -0.0005 & 1.195 \\
$1950-1960$ & +0.022 & 5.279 & +0.0696 & -0.0034 & 1.190 \\
$1960-1970$ & +0.008 & 5.250 & +0.0195 & -0.0009 & 1.197 \\
\hline
\end{tabular}

served values of $x$ and $y$. The merit of this method is to enable us to determine the values of the damping coefficient without ambiguity. These values of the constants are shown in Table I.

3. The author published a theory of the Earth's rotation, considering the variation of the interaction between the core and mantle of the Earth (Sekiguchi, 1961). According to the theory, the damping coefficient $\kappa$ and the angular velocity of the Chandlerian motion $c$ are variable and are correlated. Figure 1 gives some confirmation to the theory.

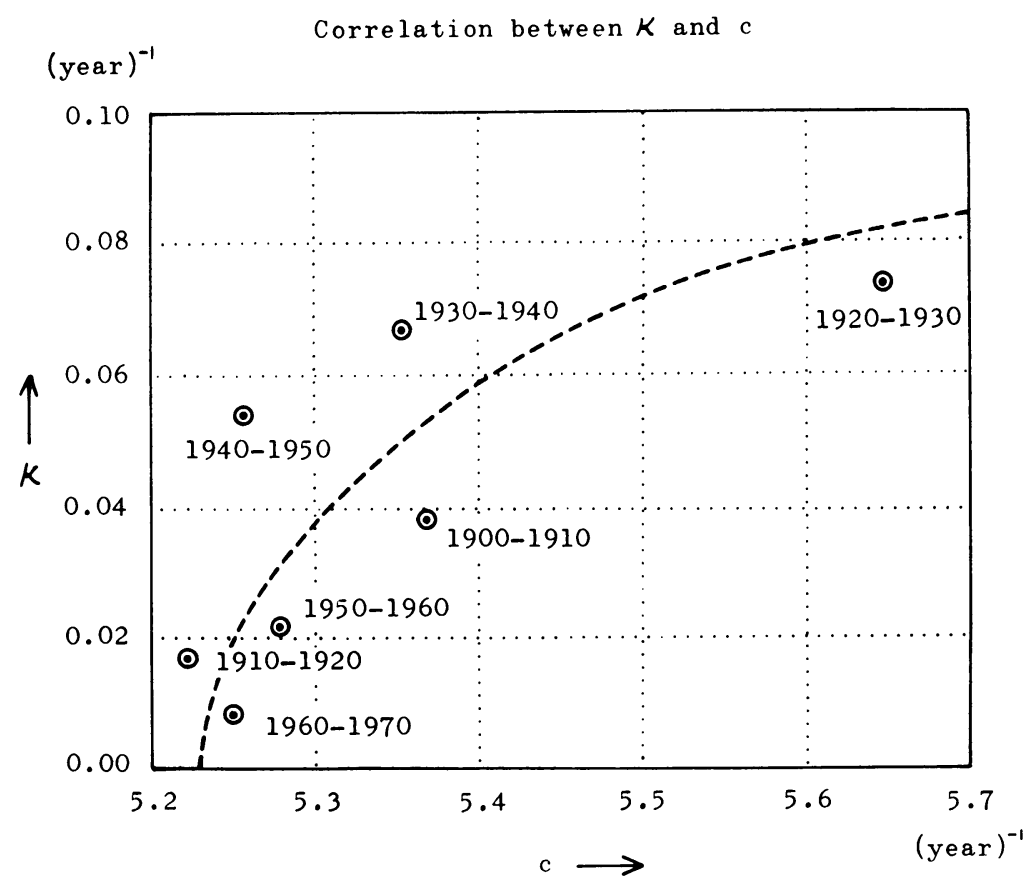

Fig. 1. 
It is well known that an increase in amplitude of the polar motion often brings about a longer Chandlerian period. But theoretically, direct correlation exists between $\kappa$ and $c$, and the large amplitude is simply the result of small $\kappa$.

4. If we assume that the excitation functions $X$ and $Y$ have form of a step function, the values of $B$ must be positive (Sekiguchi, 1966). On the other hand, if the excitation functions have the form of impulsive functions, then $B$ vanishes, and $\mu_{q}(s)$ has only a cosine term. In Figure 1, we see that the calculated values of $B$ take either positive or negative values, and we can regard $B$ as effectively zero compared with $A$. This fact suggests that the character of the excitation functions is likely to be impulsive.

\section{References}

Sekiguchi, N.: 1966, Publ. Astron. Soc. Japan 18, 116.

Sekiguchi, N.: 1961, Publ. Astron. Soc. Japan 13, 23. 